\title{
Von Lübeck zu Kaisersaschern. Die Wandlung in Thomas Manns Vorstellung von seiner geistigen Heimat
}

\author{
Liisa STEINBY \\ Universität Turku (Finland)
}

\begin{abstract}
Thomas Mann did not produce any regional literature, despite his Buddenbrooks being a Lübeck novel. In the essay Bilse und ich (1906), he explains that Lübeck was used only as a material to which he assigned symbolic meaning for creating a work of art. However, in the essay Lübeck als geistige Lebensform (1926) Mann writes that Lübeck is the growing ground of his entire artistic work. Representing an ethical stance on life, it stands for Lebensbürgerlichkeit, i.e. a commitment to the responsibilities of life. This is in accordance with how Mann, in his Betrachtungen eines Unpolitischen (1918), describes the Germans as being positioned in the "middle ground" where the demands of the intellect, or spirit (Geist), and the unconscious urges of life attain a balance. In Joseph und seine Brüder, rooted intellectually in the epoch of the Weimar Republic, the idea of Lebensbürgerlichkeit
\end{abstract}

can be recognized in a biblical setting. However, in Doctor Faustus (1947), which mirrors the epoch of the Third Reich, it is Kaisersaschern, the protagonist's (fictional) school town that represents the Germans' intellectualspiritual stance on life, which Mann now describes as an "antiquatedness of the soul". In this "soul", the irrational forces of life are uncontrollable. In the essay Deutschland und die Deutschen (1945), Mann describes Lübeck by quotations taken from the description of the medieval atmosphere of Kaisersaschern. Lübeck no longer represents the Lebensbürgerlichkeit but has become a representative of what is questionable ("demonic") in the Germans.

Keywords: Thomas Mann, Heimat, mythification

(c) Liisa Steinby; liisa.steinby@utu.fi

Colloquium: New Philologies, Volume 6, Issue 1 (2021)

doi: 10.23963/cnp.2021.6.1.4

Stable URL: https://colloquium.aau.at/index.php/Colloquium/article/view/148

This work is licensed under a Creative Commons Attribution 4.0 International License (CC BY 4.0). 
Thomas Mann hat keine Heimatliteratur hervorgebracht; auch der Lübeck-Roman Buddenbrooks ist das nicht. In seinem Aufsatz Bilse und ich (1906) weist Thomas Mann die Vorwürfe zurück, er habe in Buddenbrooks seine Landsleute karikiert und rücksichtslos dem Hohngelächter aller Welt ausgeliefert. Er beteuert, dass es hier gar nicht um seine Lübecker Bekannten geht, sondern um eine Romanwelt, die von dem Autor selbst erschaffen worden ist. Im Aufsatz Lübeck als geistige Lebensform zwanzig Jahre später weist er aber Lübeck die Rolle der geistigen Landschaft zu, die er nie zurückgelassen hat. Diese ist ein symbolhaftes, mythisches Lübeck - im Thomas-Mann'schen Sinne des Mythos zu der er sich jetzt bekennt. Im Aufsatz Deutschland und die Deutschen von 1945 gibt er Lübeck als geistiger Lebensform jedoch einen neuen Inhalt. Diese Änderungen haben nicht nur mit der Entwicklung von Manns künstlerischem Denken zu tun, sondern auch mit den historischen Veränderungen in Deutschland vom Fin de Siècle des Kaiserreichs über die Weimarer Republik bis hin zum Dritten Reich. Im vorliegenden Aufsatz gilt es, die Grundlinie dieser Entwicklung in Thomas Manns Ansicht von seiner geistigen Heimat, dem symbolisch-mythischen Lübeck, zu skizzieren.

\section{Bilse und ich: Nicht von euch ist die Rede}

„Heimatliteratur“, sowohl eine treue Darstellung des Lebens an einem Ort als auch eine satirische, sah Thomas Mann als minderwertig an (siehe Wysling 1967, 180), ja als Ausgeburt eines künstlerischen Missverständnisses. In der Rede über Lessing (1929) zitiert er zustimmend Lessing, der sich „lustig über den Provinzialismus gewisser Sittenkomödien [macht], deren Verfasser die armseligen Gewohnheiten des Winkels, in dem er geboren worden, für die eigentlichen Sitten des gemeinschaftlichen Vaterlandes halten möchte, während doch niemandem daran liege, ,zu erfahren, wievielmal im Jahre man da oder dort grünen Kohl esse“" (Mann 1990 IX, 213). Genau so, als ob es ihm um die „Gewohnheiten des Winkels“ ginge, lesen ihn aber diejenigen, die den Autor von Buddenbrooks vom „Winkel-Pasquillanten“ Bilse nicht unterscheiden können, meint Mann in Bilse und ich (Mann 1990 X, 12-13). Er will den Lesern klar machen, dass die gegebene Wirklichkeit - das reale Lübeck mit seinen Einwohnern - für ihn bloßes Material war, so wie für Shakespeare die Charaktere und Fabeln aus seiner Bekanntschaft und aus verschiedenen schriftlichen Quellen. Denn am Stoff liegt es in der Kunst gar nicht: „Alle Objektivität, alle Aneignung und Kolportage bezieht sich allein auf das Pittoreske, die Maske, die Geste, die Äußerlichkeit, die sich als Charakteristikum, als sinnliches Symbol darbietet“ (ibid., 16-17). Alles hängt davon ab, was man aus dem Material macht, wie man den Stoff „,beseelt“ (ibid., 15). Im Prozess der „Beseelung“ bewahre die Wirklichkeit ihren Charakter nicht: Beim Verfassen von Buddenbrooks hatte „meine Vaterstadt [...] 
nicht viel Realität für mich [...]. Sie war mir, mit ihren Insassen, nicht wesentlich mehr als ein Traum, skurril und ehrwürdig, geträumt vorzeiten, geträumt von mir und in der eigentümlichsten Weise mein eigen“ (ibid., 15). Thomas Mann schließt mit der Beteuerung ab: „Nicht von euch ist die Rede, gar niemals, seid des nun getröstet, sondern von mir, von mir..." (ibid., 22).

Wenn auch „Realitätspartikel“ in einem Kunstwerk wiederzuerkennen sind, ist Kunst etwas anderes als die Darstellung faktischer Realität. Der Grund für die Wahl einer „Realitätspartikel“ zum Stoff eines Kunstwerks ist, dass der Künstler darin etwas Bedeutendes und Symbolhaftes wahrnimmt, das er für sein Werk ausnutzen kann. Hier erkennt man das Zwei-Schichten-Modell des künstlerischen Denkens Thomas Manns: Die Oberfläche bietet eine Illusion der Realität, aber unter dieser Oberfläche gestaltet sich das Symbolhafte zu bedeutenden Strukturen. ${ }^{1}$ Doch während Thomas Mann in Bilse und ich von diesem Prozess als von der "Beseelung“ des Materials durch den individuellen Künstler spricht, denkt er später, dass das Leben selbst in seiner Gestaltung mythisch-typischen Mustern folgt.

\section{Lübeck als geistige Lebensform: von Lübecker Bürgerlichkeit zu Lebensbürgerlichkeit}

Im Aufsatz Freud und die Zukunft von 1936 verortet Thomas Mann seinen „Schritt vom Bürgerlich-Individuellen zum Mythisch-Typischen“ in der Zeit vor dem Joseph-Roman (Mann 1990 IX, 493). Manfred Dierks stellt aber fest, dass der Begriff des Typus bzw. des Mythos bei Thomas Mann schon vor $1911 \mathrm{im}$ Sinne der Orientierung an einem idealen Gehalt der geschichtlichen Einzelwirklichkeit zu finden ist (Dierks 1972, 39). Dierks zeigt, dass Manns Begriff des Mythisch-Typischen seinen Grund in Schopenhauers Metaphysik hat: Sein mythischer Typus sei „die vollkommene Übersetzung der Schopenhauerschen ,Species““ (ibid., 100). Der Typus sei die Form, die der Wille, der gemeinsame, irrationale Urgrund des Lebens, in der Gestaltung des Individuellen folgt. Das Individuum ist ein Fall, ein Beispiel für den allgemeinen Typus. Thomas Mann bezieht diese auf den Menschen, den er nicht nur als ein Individuum betrachtet, sondern in dem er auch den „ideellen Gehalt“ eines Typus wahrnimmt. Eine mythische Gestalt ist für ihn der Träger einer durch die kulturelle Entwicklung ermöglichten Daseinsform, die von anderen wiederaufgenommen werden kann. Im Lessing-Essay zum Beispiel definiert Thomas Mann das Klassische durch das Mythisch-Typische:

\footnotetext{
1 Zu Thomas Manns „maskenhaftem Realismus“, in dem unter der Oberfläche eine andere, tiefere Bedeutungsstruktur besteht, siehe z.B. Kristiansen 1990.
} 
Denn es ist das Vorgebildete, die anfängliche Gründung einer geistigen Lebensform durch das Lebendig-Individuelle; es ist ein erzväterlich geprägter Urtypus, in dem späteres Leben sich wiedererkennen, in dessen Fußstapfen es wandeln wird - ein Mythus also, denn der Typus ist mythisch, und das Wesen des Mythus ist Wiederkehr, Zeitlosigkeit, Immer-Gegenwart. [...] Klassische Zeit, das ist Patriarchenzeit, mythische Zeit, Zeit anfänglicher Gründung und Prägung des nationalen Lebens. (Mann 1990 IX, 229)

Das Mythische hat für Thomas Mann also nichts mit dem Magisch-Mystischen zu tun. Es ist eine kulturelle Form, die als „uranfänglich“ angesehen wird und die von neuen Individuen immer wieder aufgenommen und als deren Existenzform erlebt wird.

Was bedeutet das Mythisch-Typische aber in Bezug auf Örtlichkeiten? Wie wird ein Ort - hier: die Heimat - mythisch? Das wird ein Ort, wenn er nicht mehr als eine „geschichtliche Einzelwirklichkeit“ wahrgenommen wird, die aus einmaligen „Realitätspartikeln" besteht, sondern von seinem ,,ideellen Gehalt" her. Und dieser Gehalt ist nicht etwas, was der individuelle Autor durch die „Beseelung“ den „Realitätspartikeln“ zuweist, sondern er ist eine geistige Lebensform, die die individuelle Ortschaft exemplarisch vertritt und die als solche denjenigen, der in dieser Lebensform heimisch ist, lebenslang prägt (oder prägen kann). In Lübeck als geistige Lebensform hat Thomas Mann den Schritt von der "geschichtlichen Einzelwirklichkeit" einer Ortschaft, so wie Lübeck in Bilse und ich nur noch als Quelle des Stoffs für künstlerisches Schaffen erschien, zu einer mythischtypischen Auffassung von ihr getan.

Lübeck als geistige Lebensform ist ein Vortrag, gehalten 1926 vor einem Lübecker Publikum. Darin geht es nicht mehr um die Zurückweisung des Vorwurfs einer Verleumdung seiner ehemaligen Mitbürger. Thomas Manns Schaffen umfasste damals schon außer Buddenbrooks auch den Zauberberg, und die im Vortrag beschriebene geistige Landschaft gilt zweifellos auch für den Joseph-Roman, den er am Ende desselben Jahres niederzuschreiben begann (siehe Heftrich 1990, 449). Also nicht allein Buddenbrooks vertritt die „geistige Lebensform“ Lübecks, zu der sich der Autor jetzt bekennt. Statt einer Gegenüberstellung zwischen den Stadtbewohnern und der Freiheit des Autors, der sie als Material für sein Werk verwendet, sieht er nun eine Kontinuität zwischen der geistigen Atmosphäre der Heimatstadt und seinem künstlerischen Schaffen. Er schreibt, dass er „viel mehr ein Apfel vom Baume Lübecks war, als [er] geahnt hatte“ (Mann 1990 XI, 385). Jetzt meint er, dass die Heimatstadt „,von Anfang bis zu Ende in meiner ganzen Schriftstellerei zu finden ist, sie entscheidend bestimmt und beherrscht“ (ibid., 388). Künstlertum ist nichts völlig Ungebundenes, Freischwebendes, sondern es sei „etwas Symbolisches. Es ist die Wiederverwirklichung einer ererbten und blutsüberlieferten [!] Existenzform auf anderer Ebene“ (ibid., 385); „man hört nicht auf, zu sein, was die Väter 
waren, sondern ist ebendieses in anderer, freierer, vergeistigter, symbolisch darstellender Form nur noch einmal“ (ibid., 386). Die Heimatstadt ist nicht mehr der "Traum“ des Künstlers, sondern eine in seiner Tätigkeit auf einer ideellen Ebene fortwirkende, seine Lebenseinstellung wesentlich formende Macht.

Was ist denn das spezifisch Lübeckische in der geistigen Atmosphäre der Stadt? Thomas Mann stimmt Josef Nadler zu, wenn dieser das Lübeckische als „das PatrizistischStändische“ und „das allgemein Hanseatische“ charakterisiert (ibid., 378). ${ }^{2}$ Mann dringt aber tiefer in das Lübeckische ein und definiert es als eine ethische Haltung. Es bedeute „des Lebens ernstes Führen“, das „,mit dem Bürgerlichen in so hohem Grad zusammenfällt"; diese Haltung haben sowohl Thomas als auch sein Bruder Heinrich von ihrem Vater geerbt (ibid., 387). Sie sei in der Tiefe der Psyche des Künstlers, im Unbewussten oder Unterbewussten, bewahrt und bestimme von dort aus sein Schaffen (ibid., 385). Hier überbrückt Thomas Mann die Kluft zwischen Bürgerlichkeit und Künstlertum, dem Kaufmännischen und dem Künstlerischen, die nicht nur in Buddenbrooks, sondern zum Beispiel auch in Tonio Kröger das zentrale Thema ist. Die dem „bürgerlichen“ Ethos entsprechende Literatur lasse sich durch die Stichworte „Norden, Ethik, Musik, Humor“ bezeichnen, im Gegensatz zu „südliche[r] Schönheitsruhmredigkeit“ (ibid., 381).

Der Gegensatz zwischen Süden und Norden soll hier nicht einfach als der zwischen Süden und Norden Deutschlands verstanden werden; es handelt sich vielmehr um eine Charakterisierung der deutschen Literatur im Gegensatz zu einer stereotypisch südeuropäischen. Als ein Beispiel dafür, wie ein Werk von spezifisch norddeutschem Charakter von der ganzen Nation als das Ihrige aufgenommen werden kann, erwähnt Thomas Mann in seinem Lessing-Aufsatz Minna von Barnhelm (Mann 1990 IX, 230). Ähnliches trifft für Thomas Manns Buddenbrooks zu, aber in einem noch weiteren Sinne, denn sein Roman wurde ein internationaler Erfolg. Thomas Mann sagt in Lübeck als geistige Lebensform, dass er nicht geträumt habe, indem er „ein nach Form und Inhalt sehr deutsches Buch gab, zugleich ein überdeutsch-europäisches Buch, ein Stück Seelengeschichte des europäischen Bürgertums überhaupt gegeben haben könnte“ (Mann 1990 XI, 383). Dabei gelte es zu bemerken, dass „wir, indem wir ,Bürgerlichkeit‘ sagen, nichts Klasseninteressenmäßiges, nichts Antisozialistisches etwa im Sinne haben“" (ibid., 397). Vielmehr sei diese Bürgerlichkeit, die den Charakter der deutschen Kultur insgesamt ausmacht, eine „Weltbürgerlichkeit“, eine „Lebensform verbunden mit der Idee der Menschlichkeit, der Humanität und aller menschlichen Bildung“ (ibid., 397). Das Ethische, das den Grundton der Lübeckischen, nördlichen, deutschen „Bürgerlichkeit“ ausmacht, sei „recht eigentlich Lebensbürgerlichkeit, der Sinn für Lebenspflichten, oh-

\footnotetext{
2 Dies bedeutet natürlich nicht, dass er mit Nadler, dem späteren geprägten Antisemiten, im Allgemeinen gleichgesinnt war.
} 
ne den überhaupt der Trieb zur Leistung, zum produktiven Beitrag an das Leben und an die Entwicklung fehlt" (ibid., 387). Die Lebensbürgerlichkeit, die im geschichtlich Einmaligen entdeckt wird, sei nicht nur für Lübeck, sondern für ganz Deutschland typisch und sei eine Lebenshaltung, die sich auch im Rahmen der Menschheitsgeschichte und der Humanität behauptet. Für dieses Zusammenfallen des Partikularen mit dem Typisch-Allgemeinen findet Thomas Mann im Aufsatz Von deutscher Republik ein Vorbild bei Novalis, der schreibt: „Alles Nationale, alles Temporale, Lokale, Individuelle läßt sich universalisieren und so kanonisieren und allgemein machen [...]. Dieses individuelle Kolorit des Universellen ist sein romantisches Element“" (Mann 1990 XI, 815). Solch ein Begriff des Romantischen lässt sich von Manns „,maskenhaftem Realismus“ kaum unterscheiden: Das Konkret-Individuelle ist da, aber es wird nur bedeutend, wenn in ihm das Typisch-Allgemeine wahrgenommen wird.

Ein Beispiel dafür, wie etwas Konkretes als etwas Mythisches und Metaphysisches gedeutet wird, bieten Thomas Manns Überlegungen in Lübeck als geistige Lebensform zur Präsenz der Lübecker Landschaft in seinen Werken. Er beschreibt seine kindliche Faszination vom Meer bei sommerlichen Aufenthalten in Travemünde und kommentiert dazu:

Das Meer ist keine Landschaft, es ist das Erlebnis der Ewigkeit, des Nichts und des Todes, ein metaphysischer Traum, und mit den luftverdünnten Regionen des ewigen Schnees [d.h., der Landschaft im Zauberberg] steht es sehr ähnlich. Meer und Hochgebirge sind nicht ländlich, sie sind elementar im Sinne letzter und wüster, außermenschlicher Großartigkeit. (Mann 1990 IX, 394)

Und der unheldenhafte Held Hans Castorp zeige seine ethische Haltung und „Lebensbürgerlichkeit“, indem er angesichts der elementaren Größe der Gebirgsnatur zwar „Furcht, Ehrfurcht meinetwegen, religiöse Scheu, physisch-metaphysisches Grauen“ empfindet, aber auch „noch etwas mehr: Spott, wirkliche Ironie gegenüber dem übergewaltig Dummen, ein mokantes Achselzucken angesichts gigantischer Mächte, die ihn in ihrer Blindheit zwar physisch vernichten können, denen er aber noch im Tode menschlichen Trotz bieten würde“ (ibid., 395). Wir sind von der konkreten Landschaft des Meeres und des Hochgebirges zu deren metaphysischer und ethischer Bedeutung gekommen: zu der Herausforderung, die das Elementare für den ethisch gesinnten Menschen bedeutet. Auch wenn Thomas Mann sagt, dass ,das Meer, sein Rhythmus, seine musikalische Transzendenz auf irgendeine Weise überall in [s] einen Büchern gegenwärtig“ sei, spricht er vom Meer in einer symbolischen Bedeutung (ibid., 389). Diese Art und Weise, die Landschaft seiner Heimat als „Atmosphäre“ und Grundton seines Werkes zu bewahren, gehöre zu seinem Dasein als Künstler (ibid., 389). 
Die „Lebensbürgerlichkeit“, die in der Kunst in einem vergeistigten und erhöhten Sinn vorkommt, wird von Thomas Mann durch die Idee der Mitte beleuchtet: „Es ist die Idee der Mitte, das ist aber eine deutsche Idee. Das ist die deutsche Idee [...]. Ja, wer Deutschtum sagt, der sagt Mitte: wer aber Mitte sagt, der sagt Bürgerlichkeit“ (ibid., 396). In den Betrachtungen eines Unpolitischen (1918), die als Reaktion auf den Ausbruch des Ersten Weltkriegs entstanden, tritt die Idee der Mitte in der Form von Vermittlung zwischen „Leben“ und „Geist“, d.h. zwischen dem Unbewusst-Triebhaften oder Ursprünglich-Irrationalen und dem Geistig-Intellektuellen im Menschen, in Erscheinung (Mann 1990 XII, z.B. 25, 84, 92-93, 572-73). Die Stellung in dieser Mitte sei für die deutsche Kultur charakteristisch, und Thomas Mann entwickelt weiter den schon geläufigen Gegensatz zwischen deutscher Kultur und französischer „Zivilisation“: Im Kern der ersteren sieht er die aus dem Unbewussten schöpfende Kunst, während in der letzteren die Politik vorherrsche und das Irrationale im Leben nicht genügend berücksichtigt werde (ibid., 31-32). ${ }^{3}$ Wenn dazu noch die russische Kultur als gefühlsbetont und zum Mystizismus neigend hinzugefügt wird, also als eine Kultur, in der das Unbewusst-Triebhafte überwiegt ${ }^{4}$, fällt die geographische Mittellage der Deutschen zwischen „Westen“ und „Osten“ mit der geistigen zusammen. Die Idee vom Mittlertum zwischen „Geist“ und „Leben" wird auch in Manns weiteren Schriften bewahrt, ja sie ist der eigentliche Kern seiner Schopenhauerisch-Nietzsche'schen Metaphysik des Menschen. Schon in seinen Schriften aus den 1920er-Jahren kommt dem Mittlertum aber eine zusätzliche, politische, republikanische und demokratische Bedeutung zu: Jetzt ist es nicht nur die Aufgabe des Künstlers, sondern auch die des Politikers, zwischen den Anforderungen des Geistes und den materiellen Bedürfnissen der Menschen zu vermitteln. ${ }^{5}$

Thomas Manns Ansicht von den Lübeckern und den Deutschen - oder den Franzosen und den Russen - kann als mythologisierend bezeichnet werden. „Es ist wahr“, schreibt er in den Betrachtungen, „die Völker als mythische Individuen anzuschauen ist eine primitiv-volkstümliche Anschauungsweise“; „[e] in Künstler ist [aber] vielleicht nur eben so weit Künstler und Dichter, als er dem Primitiven nicht entfremdet ist“ (Mann 1990 XII, 150-51). Thomas Mann scheint sich hier für das Recht eines Künstlers auszusprechen, mythologisierend zu verfahren. Sein Porträt des geistigen Lübecks ist my-

\footnotetext{
3 Siehe dazu auch Koopmann 1988, 80-85.

4 Siehe Mann 1990 XII, 438-441; die drei werden auf die Formel „der politische Franzose, der menschliche Russe und der wissende Deutsche" zusammengepresst (ibid., 489). Vgl. auch Von deutscher Republik (Mann 1990 XI, 835) und Verhältnis zu Wien, wo die mittlere Position als die der Form und des Lebens zwischen zwei entgegengesetzten „Toden“ dargestellt wird, dem der Aufösung der Formen und dem ihrer Erstarrung (Mann 1990 XI, 401). Zu dieser mittleren Position des Deutschen und zur Rolle des „Russischen“ (als Inbegriff des Ostens) im Zauberberg, siehe Kristiansen 1978.

5 Siehe z.B. Von deutscher Republik (1922) und Rede vor Arbeitern in Wien (1932), Mann 1990 XI.
} 
thisch, insofern als er die „geistige Form“ Lübecks als einheitlich und zeitlos bzw. uralt und anscheinend von jeder historischen Änderung unberührt darstellt: Das „Lübeckische“ fuße auf einer "tausendjährigen“ Tradition (Mann 1990 XI, 398). Diese Tradition wirke in denen, die dort aufgewachsen sind, oft unbewusst fort - aber es sei auch möglich, sich wissentlich von einer solchen Tradition loszulösen. Thomas Mann steht dafür, dass das Tradierte und Eingewurzelte in der Kultur geachtet wird, aber doch nicht so, dass man sich selber dadurch „vom Leben und der Entwicklung“ ausschließt (ibid., 387). Lebensbürgerlichkeit bedeute auch Offenheit dafür, was eine neue historische Situation von einem verlangt.

Wie Hermann Kurzke feststellt, ist Joseph und seine Brüder ein Roman, der geistig in der Epoche der Weimarer Republik verwurzelt ist, auch wenn er erst im Exil verfasst wurde (Kurzke 1983, 225). Hier lässt sich tatsächlich die Idee der Lebensbürgerlichkeit wiedererkennen, trotz des explizit Mythischen in diesem Roman, in dem Thomas Mann nach seinen eigenen Worten die Wende vom Bürgerlich-Individuellen zum MythischTypischen vollzogen hat. Joseph und seine Brüder ist in einem doppelten Sinne ein mythischer Roman: Es wird die mythische Geschichte des biblischen Joseph wiedererzählt, und der Roman handelt von einer Welt, in der die Menschen sich selbst und ihre Welt durch Mythen deuten. Zwischen den Charakteren des Romans gibt es Unterschiede darin, wie sie mit Mythen umgehen: Die Archaischsten unter ihnen machen keinen Unterschied zwischen sich selbst und dem mythischen Vorbild, während es für andere, insbesondere für die Intellektuellen wie Joseph, zwischen dem Individuellen und dem mythischen Typus einen Hiatus gibt, der es dem Individuum ermöglicht, zwischen verschiedenen mythischen Rollen zu wählen und mit ihnen zu spielen.

Das ideelle Zentrum des Romans ist aber die "Gottessorge“ in Abrahams Sippe. Der Gott des Alten Testaments erscheint hier nicht als eine unwandelbare mythische Figur, sondern als eine sich ändernde: Die „Gottessorge“ Abrahams und seiner Nachfolger besteht in der Bemühung, zeitgemäße Änderungen im Bild Gottes wahrzunehmen und bekanntzumachen. Diese Änderungen beziehen sich darauf, was Gott vom Menschen, seinem Ebenbild, verlangt. Die „Gottessorge“ ist somit eine Sorge um den Menschen, um die Bildung der Menschheit. Thomas Mann verfährt anachronistisch, wenn er nicht nur Änderungen im Gottesbild beschreibt, sondern die Patriarchen selbst als sich ihrer Arbeit an der Entwicklung des Gottesbildes bewusst darstellt und ihnen somit die moderne Idee von der Entwicklung der Menschheit zuschreibt. Thomas Mann hat diese „Verfälschung “ aber durchaus geschickt durchgeführt, so dass seinen Lesern der Anachronismus kaum auffällt. Eindeutig spricht Thomas Mann in Joseph und seine Brüder nicht nur für die Weisheit in den uralten Mythen, sondern auch für ihre Vereinbarkeit mit dem modernen, individualisierenden und historischen Denken. 
Ziel der Bildung der Menschheit ist ein Gleichgewicht zwischen den „höheren“ und den „niedrigeren“ Mächten, dem Unbewusst-Triebhaften und dem Intellektuell-Geistigen, im Menschen. Joseph ist derjenige, der von den Mächten des Oben und von denen der Tiefe gesegnet ist, aber auch er muss lernen, seine Gaben für das Wohl anderer einzusetzen: Als Stellvertreter des Pharaos sorgt er für das materielle Wohl des Volks - sowohl des Volks des Pharaos als seines eigenen. Es geht also um die Erlangung einer „Lebensbürgerlichkeit“, die den Joseph-Roman mit der Lübecker Rede, den Betrachtungen und dem Zauberberg verbindet. Das Konkrete, Lokale ist hier ein anderes, und dieses wird ebenso lebhaft und detailreich geschildert wie die Hansestadt in Buddenbrooks oder das Davoser Sanatorium im Zauberberg. In zeitlich und geographisch voneinander entfernten Welten findet Thomas Mann also die im Grunde gleiche Problematik des Lebens und dieselbe ethische Haltung der „Lebensbürgerlichkeit“.

\section{Von Lübeck zu Kaisersaschern: Ummythologisierung des Deutschen}

Stammte Joseph und seine Brüder aus der geistigen Atmosphäre der Weimarer Republik, ist Doktor Faustus (1947) ein Roman der Epoche des Dritten Reichs. Statt Lübeck wird im Doktor Faustus Kaisersaschern als der Inbegriff des Deutschen vorgeführt. Thomas Mann arbeitete am Doktor Faustus im Exil und später auch als amerikanischer Staatsbürger, aber seine geistige Heimat blieb Deutschland; nur hat sich dessen Deutung für ihn geändert. Die neue Ansicht hat er auch im Essay Deutschland und die Deutschen von 1945 zum Ausdruck gebracht.

In Deutschland und die Deutschen ist das Deutsche nicht mehr die glückliche Mitte, die in ihrer Kultur sowohl das Irrational-Triebhafte als auch das Geistige im Menschen berücksichtigt und in Einklang miteinander bringt. Es geht zwar immer noch um das Verhältnis dieser beiden, aber jetzt gelingt die Vermittlung zwischen ihnen nicht. Statt „Lebensbürgerlichkeit“, die solch ein Gleichgewicht enthält und die dafür notwendige Weltoffenheit und Offenheit für historische Änderungen besitzt, findet Thomas Mann jetzt im Wesen der Deutschen eine Kluft. Er spricht von ihrer „Weltfremdheit“, ihrem „Weltungeschick“ und ihrem „spießbürgerliche[n] Universalismus, einem Kosmopolitismus in der Nachtmütze sozusagen“, und fügt hinzu: „Diesem Seelenbild, dieser unweltlichen und provinziellen deutschen Weltbürgerlichkeit hat immer etwas Skurril-Spukhaftes und Heimlich-Unheimliches, etwas von stiller Dämonie angehaftet, das zu empfinden meine persönliche Herkunft mir ausnehmend behilflich gewesen sein mag" (Mann 1990 XI, 1129). 
Der Autor kommt daraufhin vom „alten Lübeck“ zu sprechen, dessen Rathaus in dem Jahr vollendet wurde, ,als Martin Luther seine Thesen anschlug ans Tor der Schloßkirche von Wittenberg“(ibid., 1129-1130):
Aber wie Luther, der Reformator, nach Denkungsweise und Seelenform zum guten Teil ein mittelalterlicher Mensch war und sich zeit seines Lebens mit dem Teufel herumschlug, so wandelte man auch in dem protestanti- schen Lübeck, sogar in dem Lübeck, das ein republikanisches Glied des Bis- marck'schen Reiches geworden war, tief im gotischen Mittelalter [...]. Nein, in der Atmosphäre selbst war etwas hängengeblieben von der Verfassung des Menschengemütes - sagen wir: in den letzten Jahrzehnten des fünf- zehnten Jahrhunderts, Hysterie des ausgehenden Mittelalters, etwas von la- tenter seelischer Epidemie. Sonderbar zu sagen von einer verständig-nüch- ternen modernen Handelsstadt, aber man konnte sich denken, daß plötz- lich hier eine Kinderzug-Bewegung, ein Sankt-Veits-Tanz, eine Kreuzwun- der-Exzitation mit mystischem Herumziehen des Volkes oder dergleichen ausbräche, - kurzum, ein altertümlich-neurotischer Untergrund war spür- bar, eine seelische Geheimdisposition, deren Ausdruck die vielen „Origina- le“waren. (ibid., 1130)

Diese Beschreibung der Atmosphäre Lübecks, in der Kinderzug-Bewegung, Sankt-VeitsTanz und Kreuzwunder-Erscheinungen ihren Platz haben, ist ein Selbstzitat aus der Beschreibung Kaisersascherns, der Schulstadt und geistigen Heimat Adrian Leverkühns in Doktor Faustus (Mann 1990 VI, 52). Das „kaisersaschernisierte“ Lübeck ist nicht mehr die Hansestadt mit ihrer ethischen Haltung und der Atmosphäre einer weltoffenen Bürgerlichkeit; immer noch vertritt Lübeck das Deutsche, aber dieses wird nun wesentlich anders gedeutet. Statt der heilsamen „Mitte“, einer Position der „Lebensbürgerlichkeit“, weist der Autor von Deutschland und die Deutschen den Deutschen eine „Altertümlichkeit der Seele“ zu, „welche sich den chthonischen, irrationalen und dämonischen Kräften des Lebens, das will sagen: den eigentlichen Quellen des Lebens nahe fühlt und einer nur vernünftigen Weltbetrachtung und Weltbehandlung die Widersetzlichkeit tieferen Wissens, tieferer Verbundenheit mit dem Heiligen bietet" (Mann 1990 XI, 1143). Wo der Blick in die „Tiefe“ früher als eine Breite der Ansicht vom Menschen geschätzt wurde, wird jetzt seelische Altertümlichkeit und eine bedenkliche Nähe zum irrationalen Urgrund des Lebens, ja zum Dämonischen, gesehen.

Die Schlüsselfigur dieser Atmosphäre ist Luther, eine „riesenhafte Inkarnation deutschen Wesens" (ibid., 1132), der das Mittelalter samt dessen Dämonologie in der Moderne bewahrt. Thomas Mann gesellt sich zu Nietzsche, wenn er in der Reformation die 
Restauration des Veralteten und in Luther „ein[en] konservative[n] Revolutionär[en]“ sieht: „Er stellte ja nicht nur die Kirche wieder her; er rettete das Christentum. [...] In dem Deutschen Luther nahm das Christentum sich kindlich und bäuerlich tiefernst zu einer Zeit, als es sich anderwärts nicht mehr ernst nahm“ (ibid., 1133). Das Dämonische bei Luther und in der deutschen Kultur wird folgendermaßen geschildert: „Wo der Hochmut des Intellektes sich mit seelischer Altertümlichkeit und Gebundenheit gattet, da ist der Teufel. Und der Teufel, Luthers Teufel, Faustens Teufel, will mir als eine sehr deutsche Figur erscheinen, das Bündnis mit ihm, die Teufelsverschreibung [...] als etwas dem deutschen Wesen eigentümlich Naheliegendes“ (ibid., 1131). Das Zitat zeigt die unmittelbare Nähe von Deutschland und die Deutschen zum Doktor Faustus.

In Adrian Leverkühns geistiger Existenz und künstlerischem Schaffen setzt sich die geistige Lebensform Kaisersascherns fort, genauso wie die Lübecks in Thomas Manns, und beide vertreten dadurch das Deutsche und die deutsche Kultur. ,Wo ich bin, da ist Kaisersaschern“, könnte Leverkühn von sich sagen, ${ }^{6}$ genauso wie Thomas Mann bei seiner Ankunft auf dem Neuen Kontinent „Wo ich bin, da ist Deutschland“, gesagt haben soll. ${ }^{7} \mathrm{Da}$ „Lübeck“ eine Verwandlung durchlaufen hat und sich jetzt von Kaisersaschern als „geistiger Lebensform“ nicht unterscheiden lässt, gilt das, was Thomas Mann in der Gestalt Leverkühns von der deutschen Kultur sagt, auch für ihn selbst. Es handelt sich in Thomas Manns großem Deutschland-Roman also um eine Selbstreflexion, Selbstprüfung und Selbstkritik.

Das fiktive Kaisersaschern ist in der „Luther-Gegend“ von Halle, Wittenberg, Naumburg gelegen, was zur „Luther-Atmosphäre“ des Romans beiträgt. Hans Rudolf Vaget meint aber, dass die Schlüsselfigur im Roman nicht Luther, sondern Kaiser Otto III. sei, der in Kaisersaschern begraben ist (statt Aachen, wo seine Grabstätte eigentlich liegt) und dadurch der Stadt ihren Namen gegeben hat (Vaget 1977). In der Regierungszeit dieses Kaisers, der im Jahre 996 fünfzehnjährig zum Kaiser des Heiligen Deutsch-Römischen Reiches gekrönt wurde und schon mit einundzwanzig verstarb, verlagerte sich der Schwerpunkt der Herrschaft des Kaiserreichs nach Italien. Nach Zeitblom, dem Biographen Leverkühns und dem Erzähler des Romans, nannte der Kaiser sich „Imperator Romanorum und Saxonixus“ und war ein „Musterbeispiel der deutschen Selbst-Antipathie“ (Mann 1990 VI, 51; Vaget 1977, 220). Vaget sieht hier die positive Möglichkeit einer Selbstüberwindung des rein Deutschen und eine Anlage zum Universalismus und argumentiert dafür, dass eher Otto III. als der „mittelalterliche“ Luther die „geistige Lebensform“ Kai-

\footnotetext{
6 Dies ist ein Vorschlag des Teufels, des Alter Egos Leverkühns, im großen „Teufelsgespräch“ in der Mitte des Romans, siehe Mann 1990 VI, 302.

7 „Where I am, there is Germany“; „Mann Finds U.S. Sole Peace Hope“, in: New York Times, 22. Februar 1938, 13. Siehe dazu Koopmann 1981, 104.
} 
sersascherns vertritt, die als Erbe auf Leverkühn übergeht (ibid., 220-221). In Deutschland und die Deutschen spricht Thomas Mann von dem „Deutsche[n] in Reinkultur, $\mathrm{d}[\mathrm{em}]$ Separatistisch-Antirömische[n], „Anti-Europäische[n]“, das ihn an Luther ängstigt (Mann 1990 XI, 1133). Die Überwindung des Luther'schen könnte somit einen Durchbruch zur allgemeinen „Lebensbürgerlichkeit" und zur Position der „Mitte“ bedeuten, die nach Manns früherer Ansicht das Deutsche charakterisiert. In der Gestalt Ottos III. sieht Vaget nun Thomas Manns Stellungnahme zur Debatte deutscher Intellektueller im amerikanischen Exil über die Frage, ob der Nationalsozialismus „aus dem Wesen des Deutschtums hervorgegangen sei“ (die sogenannte Ein-Deutschland-These), oder aber ob zwischen zwei Deutschlands zu unterscheiden sei, einem guten und einem bösen (die Zwei-Deutschlands-These). ${ }^{8}$ Nach Vaget hat Thomas Mann mit der Schlüsselfigur des Kaisers Otto III. Stellung für die Ein-Deutschland-These genommen, aber in einer differenzierten Form, nämlich dass das „böse“ Deutschland das fehlgeschlagene "gute" sei; diese Ansicht wird tatsächlich in Deutschland und die Deutschen (Mann 1990 XI, 1146) vorgelegt. Dies bedeutet nach Vaget aber, dass „Thomas Manns Verständnis der deutschen Geschichte nicht in der Faust-Metapher aufgeht" und dass „[d]as gängige Interpretationsschema, wonach Leverkühn als die Verkörperung derjenigen geistigen und psychologischen Anlagen des Deutschtums aufzufassen sei, die in der Chiffre Luther und Faust gemeint seien, [...] auf einem Mißverständnis [beruht] "(Vaget 1977, 216, 220). Denn sowohl das „gute“ als auch das „böse“ Deutschland seien im Erbe Leverkühns als Möglichkeiten enthalten; er verfehle aber die Chancen, das erstere zu verwirklichen (ibid., 216-229). Vagets Argumentation reicht m. E. aber nicht dazu, das von vielen Forschern entdeckte Unvermeidliche und Schicksalhafte in Leverkühns Faust-Identifikation zu widerlegen. ${ }^{9}$ Selbst die Tatsache, dass jede eventuelle Bemühung Ottos III. zur Überwindung des rein Deutschen durch seinen frühen Tod im Keime erstickt wurde, ließe sich ihn eher als ein Symbol des notwendigen Scheiterns solcher Bemühungen der Deutschen interpretieren.

Schon in Lübeck als geistige Daseinsform wurde Musik als zum Deutschtum gehörig dargestellt. In Deutschland und die Deutschen wird als drittes Glied des Komplexes das Dämonische erwähnt:

Die Musik ist dämonisches Gebiet [...]. Sie ist christliche Kunst mit negativem Vorzeichen. Sie ist [...] die der Wirklichkeit fernste und zugleich die passionierteste der Künste, abstrakt und mystisch. Soll Faust der Repräsen-

\footnotetext{
8 Vaget 1977, 206-7. Siehe auch Vaget 1991, Vaget 2008.

9 „Bis zuletzt sieht Thomas Mann also die deutsche Geschichte, ja Geschichte überhaupt, als einen schicksalhaften Ablauf, den man nicht ändern, sondern dessen Tragik man einzig beklagen kann." (Wißkirchen 1986, 166) Vgl. Straßner $(2010,286)$.
} 
tant der deutschen Seele sein, so müßte er musikalisch sein, denn abstrakt und mystisch, das heißt musikalisch, ist das Verhältnis des Deutschen zur Welt. (Mann 1990 XI, 1131-1132)

Hier ist die Gestalt des genialen Komponisten Adrian Leverkühn vorgezeichnet, der einen Pakt mit dem Teufel eingeht, um seine Kreativität von der Lähmung zu befreien, die sein kritischer Intellekt verursacht hat. Der Teufel vertritt hier die niederen Seelenkräfte des Menschen, vor allem die Sexualität. Der Pakt mit dem Teufel wird durch Leverkühns Beischlaf mit einer Prostituierten geschlossen, bei der er sich mit einer cerebralen Syphilis infiziert. Nach fünfundzwanzig produktiven Jahren fällt er in geistige Umnachtung - im Rahmen seines Faust-Schicksals gedeutet wird er vom Teufel geholt. Die heilbringende Mitte, die Vermittlung zwischen dem Geistigen und dem Leiblich-Triebhaften, ist nicht erreicht.

Der Untergang Leverkühns steht für den Sturz der deutschen Kultur in die Barbarei des Nationalsozialismus. Im Doktor Faustus wird die Erklärung für die deutsche Katastrophe nicht in der politischen Entwicklung oder den historischen Umständen gesucht, sondern sie wird von der unabänderlichen seelischen Verfassung, dem mythischen Wesen der Deutschen abgeleitet. Die Reformation wird nicht als eine historische „Erklärung“ für das Wesen der Deutschen geboten, sondern vielmehr ist sie ein Zeitalter, in dem das Wesen der Deutschen am deutlichsten in Erscheinung tritt. Ebensowenig - oder noch weniger - wird das Deutsche aus der historischen Situation um Jahr 1000, also der Zeit Ottos III., erklärt, sondern dieser Kaiser tritt nur als ein Symbol des Deutschen in Erscheinung.

Ebenso wie Lübeck und Kaisersaschern ist Deutschland für Thomas Mann im Doktor Faustus vor allem eine geistige Größe. Die deutsche Katastrophe wird nicht durch eine historische Analyse der Gründe, sondern aus dem mythischen Charakter der Deutschen heraus erklärt. Die Unüberbrückbarkeit der Kluft zwischen dem mythischen und dem modern-aufgeklärten, historischen Denken wird in der Erzählstruktur des Romans durch die Gegenüberstellung der Perspektiven der beiden Hauptpersonen inszeniert. Leverkühn vertritt das mythische Denken, insofern als er sein Leben als Wiederholung des Lebens von Faust versteht, während Zeitblom, der an einer realistischen Künstlerbiographie schreibt, sich auf die Seite der modernen Rationalität stellt. Adrian identifiziert sich zuerst spielerisch mit Faust, aber die Identifizierung wird immer stärker, bis sie vollkommen ist. Zeitblom ist zu entsetzt und erschrocken von der Faust-Identifikation seines Freundes, um sich ernsthaft mit dieser zu befassen. Die Vorstellungen der beiden Freunde von Leverkühns Leben bleiben voneinander getrennt. Ebenso hilflos wie dem FaustSchicksal seines Freundes steht Zeitblom dem „Bösen“ im gegenwärtigen Deutschland gegenüber. Seine humanistische Idee ist die der „menschlichen Mitte“, die Domestizie- 
rung des Triebhaft-Urgründigen im Menschen durch den Geist. Sie erweist sich aber als seicht, falsch und lächerlich. Im Portrait von Zeitblom gibt Thomas Mann schweren Herzens seine frühere Idee der Humanität als Velleität auf. Eindeutig ist es Leverkühn, der die tiefere Einsicht in das Wesen des Menschen hat. Thomas Mann endet im Doktor Faustus damit, dem urtümlichen Denken in Mythen, vertreten von dem syphilitischen Künstler, rechtzugeben.

Lübeck und Kaisersaschern sind zwei Ortschaften, die Thomas Mann nacheinander als seine geistige Heimat angibt. Mit der Verurteilung der "geistigen Lebensform“ Kaisersascherns, für ihn nun der Inbegriff Deutschlands, als tief bedenklich, ja dem Bösen verfallen, verurteilt Mann auch sich selbst. Dass er als Deutscher des Deutschtums und des deutschen Schicksals unvermeidlich teilhaftig ist, hat er in Deutschland und die Deutschen explizit ausgesprochen (ibid., 1128). In der Entstehung des Doktor Faustus berichtet Thomas Mann von seiner Lungenkrankheit und Operation während der Arbeit an Doktor Faustus, die er im höchsten Maß als zur Sache gehörig ansah: „[D]er schreckliche Roman zusammen mit den deutschen Ärgernissen“ seien an seiner Erkrankung schuld (Mann 1990 XI, 254). Es ist eine Art Inszenierung mythischer Identifikation, wenn er am eigenen Leib den Zusammenbruch Deutschlands mit dem Nationalsozialismus spürt, weil er an diesem das Deutschland wiedererkennt, an dem er teilhat.

\section{Literatur}

Dierks, Manfred. 1972. Studien zu Mythos und Psychologie bei Thomas Mann. An seinem Nachlaß orientierte Untersuchungen zum „Tod in Venedig“, zum „Zauberberg“"und zur „Joseph“-Tetralogie. Thomas-Mann-Studien 2. Bern und München: Francke.

Heftrich, Eckhard. 1990. „Joseph und seine Brüder“. In Thomas-Mann-Handbuch, herausgegeben von Helmut Koopmann, 447-474. Stuttgart: Kröner.

Koopmann, Helmut. 1981. „Das Phänomen der Fremde bei Thomas Mann. Überlegungen zu dem Satz: ,Wo ich bin, ist die deutsche Kultur“". In Leben im Exil. Probleme der Integration deutscher Flüchtlinge im Ausland 1933-1945, herausgegeben von Wolfgang Frühwald und Wolfgang Schieder, 103-114. Hamburg: Hoffmann und Campe.

Koopmann, Helmut. 1988. Der schwierige Deutsche. Studien zum Werk Thomas Manns. Tübingen: Niemeyer.

Kristiansen, Børge. 1978. Unform - Form - Überform. Thomas Manns Zauberberg und Schopenhauers Metaphysik. København: Akademisk Forlag.

Kristiansen, Børge. 1990. „Das Problem des Realismus bei Thomas Mann. Leitmotiv - Zitat - Mythische Wiederholungsstruktur“. In Thomas-Mann-Handbuch, herausgegeben von Helmut Koopmann, 823-835. Stuttgart: Kröner.

Kurzke, Hermann. 1983. „Dichtung und Politik im Werk Thomas Manns von 1914-1955, Teil II“. Literatur in Wissenschaft und Unterricht 16: 225-43. 
Mann, Thomas. 1990. Gesammelte Werke in dreizehn Bänden. Frankfurt am Main: Fischer Taschenbuch Verlag.

Straßner, Frank-Uwe. 2010. Gegenwart und Gegenwelten im Deutschlandbild Thomas Manns. Frankfurt am Main: Peter Lang.

Vaget, Hans Rudolf. 1977. „Kaisersaschern als geistige Lebensform. Zur Konzeption der deutschen Geschichte in Thomas Manns Doktor Faustus." In Der deutsche Roman und seine historischen und politischen Bedingungen, herausgegeben von Wolfgang Paulsen, 200-235. Bern und München: Francke.

Vaget, Hans Rudolf. 1991. „Germany: Jekyll and Hyde. Sebastian Haffners Deutschlandbild und die Genese von Doktor Faustus." In Thomas Mann und seine Quellen. Festschrift für Hans Wysling, herausgegeben von Eckhard Heftrich und Helmut Koopmann, 249-271. Frankfurt am Main: Klostermann.

Vaget, Hans Rudolf. 2008. „Fünfzig Jahre Leiden an Deutschland. Thomas Manns ,Doktor Faustus' im Lichte unserer Erfahrung (2001).“ In Thomas Mann. Neue Wege der Forschung, herausgegeben von Heinrich Detering und Stephan Stachorski, 177-201. Darmstadt: Wissenschaftliche Buchgesellschaft.

Wißkirchen, Hans. 1986. Zeitgeschichte im Roman. Zu Thomas Manns Zauberberg und Doktor Faustus. Thomas-Mann-Studien 6. Bern: Francke.

Wysling, Hans. 1967. „,Geist und Kunst‘. Thomas Manns Notizen zu einem ,Literatur-Essay“' In Quellenkritische Studien zum Werk Thomas Manns, herausgegeben von Paul Scherer und Hans Wysling, 123-227. Thomas-Mann-Studien 1. Bern u. München: Francke. 